\title{
Recurrent sarcoid granulomas in a transplanted lung derive from recipient
} immune cells

\author{
N. Milman*, C.B. Andersen", C.M. Burton* and M. Iversen*
}

ABSTRACT: From 1992-2004, single lung transplantation has been performed in seven patients with end-stage pulmonary sarcoidosis at the Danish National Centre for Lung Transplantation. The objective was to assess whether recurrent sarcoid granulomas in the lung graft are derived from recipient or donor immune cells.

Three patients had sarcoid recurrence in the lung graft, but none had clinically overt extrathoracic sarcoidosis. Graft sex-mismatch was present in one patient, a 52-yr-old female having a lung graft from a male donor. In order to discriminate between recipient and donor cells fluorescence in situ hybridisation (FISH), using probes for both X- and Y-chromosomes, was applied on transbronchial lung biopsies (TBB) from the lung graft containing sarcoid granulomas.

The recipient's explanted lung contained multiple active sarcoid granulomas. TBB from the implanted donor lung 5 months after transplantation showed sarcoid granulomas. FISH showed that the immune cells in the granulomas were X-chromosome positive and Y-chromosome negative and, therefore, were derived from the recipient.

In conclusion, the results indicate that recurrent sarcoid granulomas in the transplanted lung are derived from recipient's immune cells, having colonised the lung allograft.

KEYWORDS: Donor, granuloma, in situ hybridisation, lung transplantation, pulmonary, sarcoidosis

$\mathbf{T}$ he aetiology of sarcoidosis is unknown. Most likely, antigen(s) of infectious origin play a role in the pathogenesis of the disease, in combination with a genetic predisposition [1, 2]. The histological hallmark of sarcoidosis is the formation of noncaseating epithelioid cell granulomas resulting from antigen stimulation. Pulmonary involvement is frequent in sarcoidosis, and in the majority of patients the lung appears to be the principal target organ [3-5].

Lung transplantation has been established as an effective therapeutic procedure in terms of survival in end-stage sarcoid pulmonary disease [6]. Recurrence of sarcoidosis in the transplanted lung occurs in $\sim 50 \%$ of Caucasian patients [6]. In theory, epithelioid cell granulomas in the transplanted lung could be formed from immune cells derived either from the recipient and/or the donor. The objective of the present study was, therefore, to examine the genetic origin of recurrent epithelioid cell granulomas in the lung allograft using fluorescence in situ hybridisation (FISH) technique.

\section{PATIENT AND METHODS}

Lung transplantation has been performed at the Danish National Centre for Lung Transplantation since 1992. The immunosuppressive regimen has consisted of induction treatment with rabbit derived antithymocyte globulin or daclizumab preparations, and triple agent maintenance immunosuppression with cyclosporine A, azathioprine and low dose prednisolone of $5 \mathrm{mg} \cdot \mathrm{day}^{-1}$. Surveillance flexible bronchoscopy (FFB) with bronchoalveolar lavage (BAL) and transbronchial lung biopsy (TBB) was routinely scheduled at $0.5,1,1.5,3,6,12,18$ and 24 months after transplantation. Using FFB, 3-4 TBB specimens were obtained under fluoroscopic guidance. The same pathologist examined all biopsy specimens, and acute cellular rejection was graded A0-A4 according to international guidelines [7]. Acute rejection episodes (A2-A4) were treated with intravenous methylprednisolone
AFFILIATIONS

*Dept of Medicine B, Division of Lung Transplantation, and \#Dept of Pathology, Rigshospitalet, University of Copenhagen, Copenhagen, Denmark.

CORRESPONDENCE

N. Milman

Dept of Medicine B 2142

Division of Lung Transplantation

Rigshospitalet

Blegdamsvej 9

DK-2100 Copenhagen $\varnothing$

Denmark

Fax: 4535452648

E-mail: milman@rh.dk

Received:

November 082004

Accepted after revision:

March 152005 
$1 \mathrm{~g} \cdot$ day $^{-1}$ for 3 days followed by high dose prednisolone, subsequently tapered to the maintenance dose.

From 1992-2004, a total of 379 lung transplantations (excluding seven re-transplantations) had been performed. Seven $(1.9 \%)$ patients had single lung transplantation (SLTx) due to endstage pulmonary sarcoidosis. This has been described previously in a series [6]. None of the patients had clinically overt extra-thoracic sarcoidosis. Follow-up of these seven transplanted sarcoidosis patients was a median of 31 months (range 12-136 months). One patient died 22 months after transplantation. Three patients had histological evidence of recurrent sarcoidosis in the lung allograft, and only one of these patients had radiological appearances consistent with pulmonary sarcoidosis (i.e. adenopathy and/or reticulo-nodular parenchymal infiltrates).

Graft-sex mismatch was present in one female patient having a right-sided lung allograft from a 20 -yr-old male donor " $\mathrm{M}^{\prime \prime}$. Macroscopic inspection of the donor lung at the time of surgery was unremarkable. The female recipient was 52 yrs old at the time of SLTx. The duration of sarcoidosis until SLTx was 25 yrs. Examination prior to transplantation showed chest radiograph results compatible with stage III pulmonary sarcoidosis and severely reduced lung function values: forced expiratory volume in one second (FEV1) $24 \%$ predicted, forced vital capacity (FVC) $47 \%$ pred and carbon monoxide diffusing capacity of the lung (DL,CO) 60\% pred. Functional status was New York Heart Association class III, with a 6 min walk distance of $80 \mathrm{~m}$.

Histological examination of the recipient's explanted lung revealed multiple active sarcoid granulomas. After transplantation, the peri-operative course was uncomplicated. Within 4 months after transplantation the patient had three acute cellular rejection episodes (A2-A3) treated with corticosteroids. Maximum pulmonary function was attained at 4 months post-transplant: FEV1 $66 \%$ pred, FVC $71 \%$ pred and DL,CO $67 \%$ pred. During this period, there had been no radiological or histological evidence of sarcoidosis in the donor lung. TBB from the donor lung 5 months after transplantation showed recurrence of sarcoid granulomas. At this time, chest radiographical and computerised tomography (CT) scanning of thorax demonstrated mediastinal lymphadenopathy and parenchymal nodules throughout the right lung allograft, consistent with sarcoidosis. Radiological appearances of the patient's native lung remained unchanged; specifically, there was no evidence of pulmonary sarcoidosis relapse. Immunosuppressive treatment remained unchanged. Two subsequent bronchoscopies with TBB at 6 and 9 months post-transplant, demonstrated peribronchial fibrosis without evidence of sarcoid granulomas or acute cellular rejection. A follow-up chest radiograph and $\mathrm{CT}$ scan of the thorax demonstrated progressive volume reduction and diffuse increase in parenchymal density in the lung allograft, with regression of mediastinal lymphadenopathy. In the absence of concomitant infection and/or acute rejection, a diagnosis of bronchiolitis obliterans syndrome (BOS) was made [8]. The last lung function test was severely reduced: FEV1 $21 \%$ pred, FVC $35 \%$ pred and DL,CO $37 \%$ pred. BOS was resistant to therapy and the patient died 22 months after transplantation, an autopsy was not performed.
Donor M's left lung was unsuitable for transplantation as a result of contusion injury; however, the liver was used for transplantation of a 48-yr-old male recipient with end-stage liver disease secondary to sclerosing cholangitis. The liver recipient has had no symptoms or radiological appearances consistent with sarcoidosis, and no evidence of granulomas in liver biopsies taken 2 weeks and 4 yrs after transplantation.

\section{HISTOLOGY AND FLUORESCENCE IN SITU HYBRIDISATION}

TBB specimens were formalin fixed and processed immediately by a microwave oven technique to paraffin, from which serial sections (3-4 $\mu \mathrm{m}$ in thickness) were cut to obtain full coverage of the tissue. Samples were stained with haematoxylin-eosin, Periodic-acid-Schiff, silver staining and MassonTrichrome. Ziehl-Nielsen staining was performed to detect mycobacteria and immunohistochemical studies were made to detect cytomegalovirus infection.

FISH was applied on TBB from the lung allograft containing sarcoid granuloma using probes for the X- and Y-chromosomes. Biocolour FISH was performed on 1-2 $\mu \mathrm{m}$ thick paraffin sections using specific $\alpha$-satellite DNA-probes for the X-chromosome (CEP®X (DXZ1, $\alpha$-satellite) and SpectrumGreen $^{\mathrm{TM}}$ (Vysis, Downers Grove, IL, USA)), and the Y-chromosome (CEP®Y (DYZ3, $\alpha$-satellite) and SpectrumOrange $^{\mathrm{TM}}$ (Vysis)) according to a standard protocol recommended by DakoCytomation (Copenhagen, Denmark), using heating in pre-treatment-solution followed by proteolytic digestion by cold ready to-use-pepsin at room temperature. Denaturation was performed for $1 \mathrm{~min}$ at $82^{\circ} \mathrm{C}$ and hybridisation took place over night at $42^{\circ} \mathrm{C}$. The number of hybridisation signals for each probe was assessed in the cells constituting the granulomas and the surrounding tissue. Normal human male and female lung tissue was used as a control.

\section{RESULTS}

Staining, PCR and culture for mycobacteria, as well as immune staining for Cytomegalovirus was negative in all TBB specimens.

In the explanted lung, the majority of cells in the sarcoid granulomas and the adjacent lung tissue displayed positive signals for the $\mathrm{X}$-chromosomes, but no signal for the $\mathrm{Y}$ chromosome (figure not shown). FISH findings in the transplanted lung are shown in figure 1. In the lung allograft, cells constituting the sarcoid granulomas display positive signals for the X-chromosomes, but no signal for the $\mathrm{Y}$ chromosome. In contrast, the adjacent lung tissue displays positive signals for both the $\mathrm{X}$ - and the $\mathrm{Y}$-chromosome.

The pattern of distribution of the $\mathrm{X}$ - and $\mathrm{Y}$-chromosome signals indicates that immune cells originating from the female recipient form the sarcoid granulomas in the transplanted lung.

\section{DISCUSSION}

The lung is the most commonly involved organ in sarcoidosis $[3,4]$. Pulmonary involvement shows regression or stability in $>90 \%$ of patients [5]. Severe pulmonary dysfunction occurs in a small percentage of the patients and accounts for most cases of morbidity and mortality $[3,5]$. 

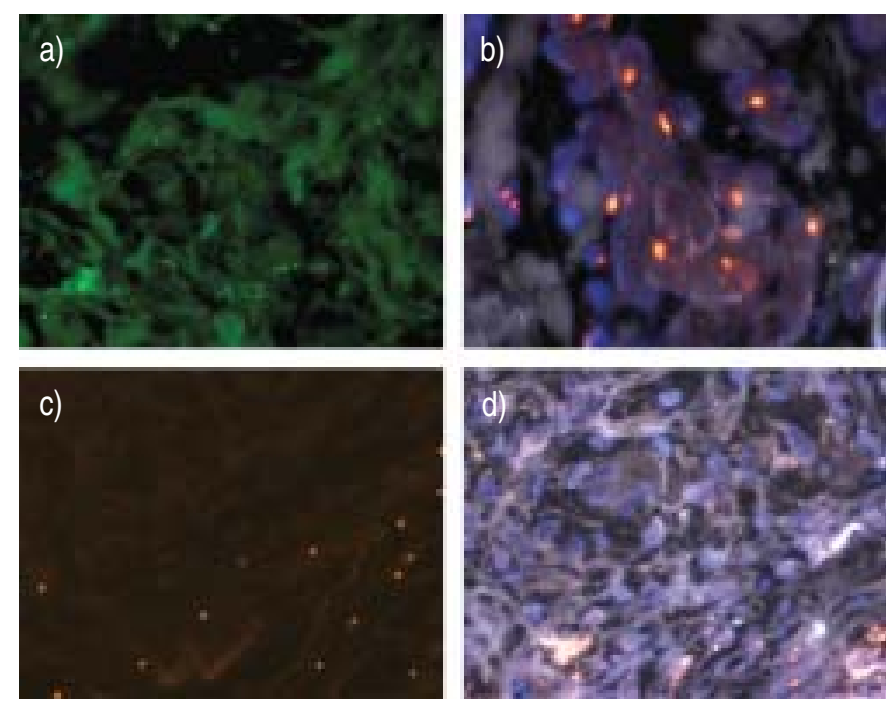

FIGURE 1. a) Fluorescence in situ hybridisation (FISH) with the X-probe (SpectrumGreen) in the centre of the granuloma demonstrating several of the epithelioid macrophages to be of female genotype with two distinct signals in their nuclei. b) FISH with the X- and Y-probe using a triple-filter (SpectrumGreen and SpectrumOrange) in the lung tissue surrounding the granuloma showing the majority of cells to be of male genotype with a large orange signal for the $Y$ chromosome and a smaller green dot for the X-chromosome. c) FISH with the $\mathrm{Y}$ probe (SpectrumOrange) demonstrating the borderline between the granuloma (upper left field) and the surrounding normal tissue (lower right field). Cells containing the Y-chromosome are situated outside the granuloma. d) FISH with both probes using a triple filter demonstrating the double $\mathrm{X}$-chromosome containing cells (green signals) to be located in the granuloma (upper left field) and the $\mathrm{X}$ - and $\mathrm{Y}$-chromosome containing cells to be located outside the granuloma (lower right field).

Following pulmonary transplantation for this condition, a diagnosis of disease recurrence in the lung allograft will, among other factors, depend on the frequency of surveillance bronchoscopy and the number of TB; i.e. in a given series, more intense surveillance will increase the number of recognised recurrences. This will impede comparison of recurrence rates in patient series having different frequencies of surveillance bronchoscopy. Appearance of granulomas in the lung allograft of recipients transplanted for pulmonary sarcoidosis occurred in three (43\%) out of the seven patients. In a series of 12 patients having ad hoc bronchoscopy, disease recurrence was observed only in three (25\%) patients [9]. Recurrence appears to be higher, $\sim 66 \%$, in African-American patients [10]. Other series have reported recurrence rates of between $33-80 \%$ [11-13].

The majority of patients with disease recurrence in the allograft have no sarcoid-related pulmonary symptoms, and recurrence does not seem to have any significant influence on lung function, at least in the short-term $[10,14]$. In this small study, only one of the three patients with recurrent sarcoidosis experienced temporally related pulmonary symptoms. In addition, a review of all thoracic radiology pertaining to the seven transplanted patients revealed that this was the only patient to have had overt radiological evidence of recurrent disease in the lung allograft.
Non-necrotising epithelioid cell granulomas are the histological hallmark of sarcoidosis. Most likely, these granulomas are formed in response to a persistent inflammatory antigen [1-3]. The dominant cellular constituents of mature epithelioid cell granulomas are non-lymphoid mononuclear cells, which include blood monocytes and tissue macrophages (histiocytes) [15]. In epithelioid cell granulomas, cells belonging to the mononuclear phagocyte system are represented by the macrophages, epithelioid cells and giant cells of the Langhans type [15]. Studies of the Kveim test show that epithelioid cell granulomas can develop in 10-12 days [16]. Accordingly, recurrent sarcoidosis in the lung allograft has been reported as early as two weeks after transplantation [13]. In the three patients within this study, recurrence was observed at 2, 5 and 6 months after transplantation, respectively [6].

It has been suggested that the evolution of allograft rejection and sarcoid granulomas may share a common pathogenesis via activated T-lymphocytes [10, 17]. However, it is conceivable that the formation of sarcoid granulomas and the mechanism of allograft rejection are governed by different immunological pathways [18]. Sarcoid granulomas are considered to be a CD4 T helper 1-type T-lymphocyte mediated process [19, 20], whereas allograft rejection displays characteristics of a lytic CD8 cytotoxic T-lymphocyte response to allograft cells $[18,21]$. These differences between the two processes are emphasised by the different responses to immunosuppressive treatment. Cyclosporine $\mathrm{A}$ is effective in controlling allograft rejection, but ineffective in controlling the clinical course of sarcoidosis [22]. The observation that recurrence of sarcoid granulomas is common in the transplanted donor lung despite treatment with cyclosporine $\mathrm{A}$, is in accordance with this interpretation.

The transplanted donor lung harbours variable amounts of immune cells (lymphocytes, monocytes, alveolar macrophages) from the donor. These cells will be exposed to the putative antigen involved in the pathogenesis of sarcoidosis, and might, in theory, respond by formation of epithelioid cell granulomas. After blood perfusion has been re-established in the transplanted lung, immune cells from the recipient colonise the graft and start to replace the donor immune cells. Although a few cases of graft versus host disease have been described in association with lung transplantation [23], in the majority of transplanted patients, the donor lymphocytes disappear from the BAL fluid within three months, and the donor alveolar macrophages disappear from the BAL fluid within 6 months of transplantation [24]. This is either due to apoptosis or due to the deleterious effect of the recipient immune response to the allograft. Donor alveolar macrophages may be the cell type with the longest "survival" time in the lung allograft, as in a few patients, they have been recovered in BAL fluid several years after transplantation [24]. In the patient in the present study, the sarcoid granulomas in the donor lung were discovered 5 months after transplantation, i.e. donor immune cells might still have been present in the lung allograft.

In theory, the donor could have had undiagnosed, subclinical pulmonary sarcoidosis. The left donor lung had a contusion and was unacceptable for transplantation; however, the donor liver was transplanted. The recipient did not show evidence of sarcoid disease and surveillance biopsies of the liver did not 
show any granulomas. Therefore, it appears unlikely that unrecognised sarcoid disease could have been transmitted with the donor lung.

The genetic predisposition involved in the pathogenesis of sarcoidosis may also influence the reaction of the immune cells following exposure to the unidentified sarcoidosis antigen. The recipient immune cells are known to carry the genetic predisposition. The donor immune cells probably do not have a similar genetic predisposition and, therefore, may not react by granuloma formation when exposed to the same antigen.

In conclusion, the present results show that recurrent sarcoid epithelioid cell granulomas in the transplanted lung allograft are derived from recipient immune cells; however, additional studies are required to substantiate these findings.

\section{REFERENCES}

1 Milman N. Is sarcoidosis an infectious disease? Review series in respiratory diseases. Stockholm. Helix Communications 1998; 1: 2-5.

2 Du Bois RM, Goh N, McGrath D, Cullinan P. Is there a role for micro-organisms in the pathogenesis of sarcoidosis? J Intern Med 2003; 253: 4-17.

3 Hunninghake GW, Costabel U, Ando M, et al. ATS/ERS/ WASOG statement on sarcoidosis. Sarcoidosis Vasculitis Diffuse Lung Dis 1999; 16: 149-173.

4 Milman N, Selroos O. Pulmonary sarcoidosis in the Nordic Countries 1950-1982. Epidemiology and clinical picture. Sarcoidosis 1990; 7: 50-57.

5 Milman N, Selroos O. Pulmonary sarcoidosis in the Nordic Countries 1950-1982. II. Course and prognosis. Sarcoidosis 1990; 7: 113-118.

6 Milman N, Burton C, Andersen CB, Carlsen J, Iversen M. Lung transplantation for end-stage pulmonary sarcoidosis: outcome in a series of seven consecutive patients. Sarcoidosis Vasculitis Dif Lung Dis 2005; 22: (In press).

7 Yousem SA, Berry GJ, Cagle PT, et al. Revision of the 1990 working formulation for the classification of pulmonary allograft rejection. J Heart Lung Transplant 1996; 15: 1-15.

8 Estenne M, Maurer JR, Boehler A, et al. Bronchiolitis obliterans syndrome 2001: an update of the diagnostic criteria. J Heart Lung Transplant 2002; 21: 297-310.

9 Walker S, Mikhail G, Banner N, et al. Medium term results of lung transplantation for end stage pulmonary sarcoidosis. Thorax 1998; 53: 281-284.

10 Nunley DR, Hattler B, Keenan RJ, et al. Lung transplantation for end-stage pulmonary sarcoidosis. Sarcoidosis Vasc Diffuse Lung Dis 1999; 16: 93-100.
11 Bjortuft O, Foerster A, Boe J, Geiran O. Single lung transplantation as treatment for end stage pulmonary sarcoidosis: recurrence of sarcoidosis in two different lung allografts in one patient. J Heart Lung Transplant 1994; 13: 24-29.

12 Burke M, Stewart S, Ashcroft T, Corbishley C, Bishop P, Kjellström C. Biopsy diagnosis of disease recurrence after transplantation (TX) for pulmonary sarcoidosis: a multicentre study. J Heart Lung Transplant 2001; 20: 154-155.

13 Johnson BA, Duncan SR, Ohori NP, et al. Recurrence of sarcoidosis in pulmonary allograft recipients. Am Rev Resp Dis 1993; 148: 1373-1377.

14 Judson MA. Lung transplantation for pulmonary sarcoidosis. Eur Respir J 1998; 11: 738-744.

15 Sheffield EA, Williams WJ. Pathology. Lung Biology in Health and Disease 1994; 73: 45-67.

16 Sheffield EA, Mitchell DN, Dewar A, Corrin B. The ultrastructural features of developing Kveim test granulomas. Sarcoidosis 1989; 6: Suppl. 1, 6-7.

17 Martinez FJ, Orens JB, Deeb M, et al. Recurrence of sarcoidosis following bilateral allogenic lung transplantation. Chest 1994; 106: 1597-1599.

18 Semenzato G, Agostini C. Lung transplantation in Sarcoidosis: lessons learned from immunology. Sarcoidosis Vasc Diffuse Lung Dis 1999; 16: 21-23.

19 Agostini C, Trentin L, Facco M, et al. Role of IL-15, IL-2 and their receptors in the development of $\mathrm{T}$ cell alveolitis in pulmonary sarcoidosis. J Immunol 1996; 157: 910-918.

20 Moller DR. Cells and cytokines involved in the pathogenesis of sarcoidosis. Sarcoidosis Vasc Diffuse Lung Dis 1999; 16: 21-23.

21 Szeto WY, Rosengard BR. Basic concepts in transplantation immunology and pharmacologic immunosuppression. In: Baumgartner WA, Reitz BR, Kasper E, Theodore J, eds. Heart and lung transplantation. 2nd Edn. London, WB Saunders Company, 2002; pp. 15-25.

22 Wyser CP, van Schalkwyk EM, Alheit B, Bardin PG, Joubert JR. Treatment of progressive pulmonary sarcoidosis with cyclosporine A. A randomised controlled trial. Am J Respir Crit Care Med 1997; 156: 1371-1376.

23 Luckraz H, Zagolin M, McNeil K, Wallwork J. Graftversus-host disease in lung transplantation: 4 case reports and literature review. J Heart Lung Transplant 2003; 22: 691-697.

24 Wiebe BM, Mortensen SA, Petterson G, Svendsen UG, Andersen CB. Macrophage and lymphocyte chimerism in bronchoalveolar lavage cells from human lung allograft recipients. APMIS 2001; 109: 435-440. 